\title{
versants
}

\section{Bandini, il dialetto, la rima ${ }^{\mathrm{I}}$}

Rodolfo ZuCCO

Università degli Studi di Udine

\begin{abstract}
Poeta fermamente convinto che compito sociale e politico precipuo dello scrittore in versi contemporaneo, soprattutto quello italiano, sia quello di affrontare e vincere il logoramento della lingua, Bandini si è tuttavia confrontato anche con la scrittura in versi dialettali, con particolare intensità nel ventennio dalla metà degli anni Settanta alla fine degli anni Novanta. Questa sperimentazione del vicentino dirama dal suo interesse per l'esplorazione dell'universale a partire da una ridotta topicità (geograficamente, Vicenza), dalla considerazione del dialetto come una forma di passato potenzialmente portatrice di «segnali per il futuro» e dalla rivisitazione, nel dialetto, dell'infanzia. Al dialetto come esperienza infantile può essere connessa l'esplorazione del dialetto come fonte di inediti accostamenti rimici.
\end{abstract}

Keywords: Bandini, primazia della scrittura in italiano, scrittura in vicentino cronologia, funzioni, rima.

I. È nota la posizione di Fernando Bandini riguardo a quello che potremmo definire il mandato politico-linguistico dello scrittore in versi in Italia. Nel 1997, concludendo il suo intervento Le ragioni della poesia, affermava:

Dialetto e latino sono lingue-rifugio, camminamenti di talpa scavati sotto la terra per vedere le parole dalla parte della radice. Ma il compito del poeta è oggi misurarsi con un limpido, saldo italiano della poesia. Hic Rhodus, hic salta.

È su quel campo, quello dell'italiano, che troveremo le nostre vittorie, se la nostra pazienza sarà stata tenace e le Muse si mostreranno benigne; è su quel campo che, diversamente, conosceremo le nostre sconfitte (Bandini I998b: 38).

Ancora dieci anni dopo, conversando con Gianfranco Bettin, Goffredo Fofi e Leonardo Ruffin, ribadiva:

Io dissuado dallo scrivere in dialetto perché mi sono accorto che da un po' di tempo il dialetto possiede una posizione di privilegio, con tendenze alla sopravvalutazione rispetto a quello che effettivamente sta producendo. In sostanza, attraverso il dialetto si sta realizzando quel tipico monolinguismo

I Tutte le citazioni da poesie di Bandini si intendono tratte da Bandini 2018. 
novecentesco che era caratteristico della poesia negli anni trenta, quaranta e cinquanta, con fughe costanti verso l'idillio (Bandini 2009: 172).

\section{E più avanti:}

quando devi usare questa lingua ingrata che è l'italiano, che poi è l'italiano continuamente attaccato e deformato dai media, riuscire, come diceva il vecchio Mallarmé, a rendere più puro il dialetto della tribù è una cosa davvero ingrata. Perché è proprio la lingua che ti sfugge: è atona, è logora, è sporca, no? (Bandini 2009: I73).

E in effetti la scrittura di versi vicentini da parte di Bandini ha tutti i caratteri dell'eccezionalità. Eccezionalità «ispirativa», innanzitutto (il dialetto può «essere considerato il recupero di una lingua magica e nativa [...] soltanto nell'eccezione della lingua della poesia come lingua morta che aveva il Pascoli, ma perché questo accada devi essere nella grazia delle Muse»: Bandini 2009: 173$)^{2}$; e poi eccezionalità tematica e cronologica. Per quella tematica, fui io stesso a raccogliere questa dichiarazione:

Ci sono dei momenti in cui il dialetto si impone in maniera imperiosa, e non c'è possibilità di scelta: cioè non ci sono cose che possono essere scritte tanto in italiano quanto in dialetto, come ahimè pensano alcuni poeti in dialetto che fanno addirittura gli sperimentali. Ecco che per me le poesie in dialetto, che pure io personalmente amo, sono sempre nate in via d'eccezione (Bandini 200I: 72$)^{3}$.

\section{Col dialetto - ha specificato Beccaria -,}

lingua della memoria infantile, Bandini fa risuonare, più che l'emozione dell'oralità o l'animato quadro d'ambiente, i lati oscuri della psiche. È la lin-

2 Si veda anche Bandini (2008b: 22): «Il dialetto rappresenta una sorta di scampo o di rifugio dalla situazione di logoramento della lingua italiana, sembra essere vicino a un mondo intatto, chiuso nella memoria, una lingua morta, quella che parlavano mio padre e mia madre». Quanto al concetto di ispirazione, si veda questo passaggio in Bandini (2006: II): «quella che i lettori "teocon" della poesia, come il sottoscritto, si ostinano a chiamare "ispirazione", è un filo unitario che continua a svolgersi dallo stesso gomitolo nell'animo del poeta. Durata e fedeltà garantiscono la coerenza di quella voce e insieme i movimenti della scrittura, comprese le novità della stessa scrittura. Intendendo per ispirazione l'insieme di pulsioni, di temi prediletti e peculiare visione del mondo, di quel particolare avatara del senso poetico che l'aristocrazia critica dei Novecentisti (i quali non si peritavano di vestire da intelligenza, con distillati e sapienti moduli stilistici, anche clandestini batticuori) usavano chiamare "messaggio"».

3 La battuta prosegue così: «anche perché il problema fondamentale della poesia, soprattutto in Italia, è riuscire ad aggredire questa lingua sempre più logorata dall'uso che ne fanno i mass-media, sempre più negletta e infarcita di forestierismi, soprattutto di anglismi. E la poesia è non soltanto fare la poesia ma anche ritrovare la lingua». 
gua che più non si sa, o meglio si sa ma non si parla («Sta lingua mi / la so ma no la parlo, / la xe lingua de morti», Santi di Dicembre, Sta lingua), una lingua intinta di mistero, quella che la nonna ha imparato dalle mitiche fate dell'acqua, le anguane, lingua pantera odorosa che è ancora per ogni dove ma non sai dove stia, si spande, egli dice, come una polvere di fieno soffiata dal vento, volando attraverso le altane. Non c'è uso imitativo o espressionistico del dialetto. Il dialetto è evocazione del mondo infantile, «residuo esistenziale», e quella parola privata inerisce profondamente alla cosa (Beccaria 20I8: XIII) ${ }^{4}$.

Quanto all'eccezionalità cronologica, laddove la scrittura in latino di Bandini copre l'arco cronologico dalla fine degli anni Cinquanta al primo decennio del nuovo secolo, la maggior parte delle poesie vicentine risalgono a un giro d'anni piuttosto circoscritto. Benché la data della «chiusura» editoriale di La mantide e la città (Bandini I979: a stampa nel marzo) non possa essere considerata con certezza un terminus post quem, tenderei a pensare che il corpus dialettale bandiniano edito nei primi due libri garzantiani sia stato composto nel ventennio che segue la pubblicazione del secondo libro5. Si tratta di venti poesie, in tutto 485 versi. Nove sono pubblicate nella sezione In lingue morte nell'"Almanacco dello Specchio» I2 (Bandini I986) e quindi accolte in Santi di Dicembre (Bandini I994): sono Imbriago, Carnevale, I puteleti del vampiro, Desso i me spoia nuo, La grande late, Vento e fogo, Drio de la porta, Sta lingua e No volevo ${ }^{6}$; undici si leggono in Meridiano di Greenwich: due - La ciupinara e El nome - nella sezione trilingue Cose del mondo di sotto, nove in quella interamente dialettale, Oga Magòga: Spassaure, Guera, L'anfelo imbissà, L'omo col saco, Oga Magòga, So' tel canton, Sagra, Casa voda, L'anguria ${ }^{7}$. Alla produzione in proprio va aggiunta la traduzione in vicentino della sestina di Arnaut Daniel (Bandini I985; ora anche in Bandini 2018: 6I6-6I7; l'incipit recita «Sta voia che me tira drento»).

Prima della pubblicazione di In lingue morte, le presenze dialettali nel corpus poetico di Bandini non toccano più di quattro testi. Il primo è Vento in Valsugana, di In modo lampante (Bandini 1962), una filastrocca di cinque

4 Il virgolettato proviene da Segre (I99I: 64). Sui temi delle poesie vicentine di Bandini $c f r$. ora Todoverto (2016/2017).

5 È auspicabile che una datazione più precisa emerga dall'esame delle carte presenti nell'Archivio di Fernando Bandini presso la Biblioteca dell'Accademia Olimpica di Vicenza. Cfr., intanto, infra.

6 Conclude la sequenza da 'Sancti duo', anticipazione del poemetto Sancti duo decembris mensis. Esso sarà ripreso integralmente nella sezione In lingue morte di Santi di Dicembre (Bandini 1994), dove, immutato l'ordine delle poesie vicentine, è collocato tra La grande late e Vento e fogo (sul portato tematico-strutturale della nuova forma della sequenza cfr. Todoverto (2016/2017: 20).

7 Di nessuna di esse risulta, allo stato attuale della ricerca bibliografica, una pubblicazione precedente. 
versi inserita (in corsivo) all'interno e in conclusione di una serie di cinque quartine di versi liberi:
Sècio, secèlo, oro pu belo, oro pu fin, secondo marìn, tre naranze, tre limoni per andare in becaria, cichete, ciòchete, volta via ${ }^{8}$.

Vento in Valsugana non sarà ripresa in Memoria del futuro (Bandini I969); tuttavia l'esordio del Bandini dialettale con una filastrocca infantile è un dato essenziale per il discorso che vorrei svolgere. Vi tornerò. Memoria del futuro riprende invece, da Per partito preso (Bandini I965), due poesie della sezione Neve e tuono (I962-I963): una interamente dialettale (tranne che nel titolo), Farfalle e vischio, e un'altra, Mia madre cuciva tomaie, in cui «il veneto è impiegato contrappuntisticamente, in integrazioni contestuali con la lingua» (Mengaldo 20I3: 253). Impiego contrappuntistico si ha anche nella poesia più tarda (nella sezione Spade di legno, con poesie del I966-68), Quello che è vietato (già in "Quaderni piacentini», V, 28, settembre I966, p. I30; cfr. Mengaldo 20I3: 253-254 e Todoverto 20I6/20I7: I4-I5). Cosicché Mengaldo potrà scrivere che «le modalità dell'uso (assai circoscritto) che Bandini fa del dialetto mostrano con esattezza, in re e in negativo, come e qualmente esso non sia utilizzabile per lui ad altri titoli e dosi» (Mengaldo 20I3: 253): dosi che, come ho anticipato, arrivano ad azzerarsi (o comunque si sottraggono a un esito pubblico) nelle scritture degli anni Settanta.

In Dietro i cancelli e altrove (Bandini 2007) l'unica presenza dialettale è la battuta della madre in conclusione di Rappresentazione della mia morte al tempo delle guerre in Medio Oriente: «Sito propio Fernando, el me putelo?». Una scelta ponderata e argomentata: nel libro «c'è solo una frase in dialetto, che è quella che mi viene rivolta da mia madre morta. Avevo un paio di poesie in dialetto ma le ho escluse apposta. Ho detto: "L'unico dialetto deve restare quello di queste parole di mia madre negli inferi" e quindi non ho messo nel libro poesie in dialetto» (Bandini 2009: 173$)^{9}$.

2. La ridotta estensione cronologica della scrittura in versi dialettale di Bandini è una diretta conseguenza del suo carattere intrinsecamente

8 Ne tratta dettagliatamente Todoverto (20I6/2017: 9-II).

9 Non sono in grado, al momento, di precisare a quali poesie Bandini faccia qui riferimento. Posso però dire che il fascicolo contrassegnato G/III/42 dell'Archivio di Fernando Bandini presso l'Accademia Olimpica di Vicenza contiene - insieme ad altre carte di lavoro relative a testi vicentini di Santi di Dicembre e di Meridiano di Greenwich e alla traduzione in vicentino di Arnaut Daniel (Bandini 1985) - venti fogli manoscritti o dattiloscritti (solo tre in pulito, gli altri con interventi manoscritti) riferibili a quattordici poesie inedite. 
sperimentale, per sperimentazione intendendosi l'atteggiamento che Bandini stesso attribuiva a Dante («È il poeta più sperimentale dell'Occidente - ha scritto -, molto più coraggioso di quanti, nel secolo che si sta per concludere, hanno sperimentato forme e modi inediti della poesia»: Bandini 2002a: I24), vale a dire la capacità di «modificare il proprio stile, facendo scelte di linguaggio a seconda del clima che attornia i propri sogni e le proprie evocazioni» (Bandini 2002a: I24). Si tratterà, dunque, di chiedersi quale clima, quali evocazioni caratterizzino il sentire di Bandini negli anni in cui si formano Santi di Dicembre e Meridiano di Greenwich in modo tale da determinare la scelta - acca nto all'italiano e al latino ${ }^{\mathrm{IO}}$ - del dialet to di Vicenza come lingua della propria poesia. Mi pare si possano individuare, come moventi della bandiniana «volontà di dire» in dialetto, tre fattori convergenti. Il primo ha come base il rapporto con la sua città: Vicenza-Aznèciv: «Sono totalmente immerso in Vicenza - ha detto -, in un rapporto di odio-amore, cerco di interpretare il mio tempo partendo da questo piccolo spazio» (Bandini 2009: I6o). È una scelta il cui valore il Bandini critico ha riconosciuto in altri poeti suoi contemporanei (si veda quanto ha scritto della poesia di Eugenio De Signoribus ${ }^{11}$, Ferruccio Benzoni ${ }^{12}$ e Paolo Bertolani) ${ }^{13}$. Per un limitato periodo di tempo, dunque, Bandini sperimenta la possibilità del confronto con la Storia a partire non solo da una «ridotta "topicità"» (Bandini I994: 94) ${ }^{14}$ fisico-geografica, ma anche nei confini di quella lingua che di questa «ridotta

Io Tra la metà degli anni Settanta e il 1997 di Meridiano di Greenwich Bandini dà alle stampe Niveus nimbus, Carmen Fernandi Bandini in Certamine poetico Hoeufftiano magna laude ornatum, in Circus equestris [Olindo Pasqualetti], Sub solis ortum [Teodoro Ciresola], Niveus nimbus, Carmina Certaminis poetici Hoeufftiani edidit Academia Regia Disciplinarum Nederlandica, Amstelodami, 1977, pp. 2I-23 (poi in plaquette: Niveus nimbus, traduzione dell'autore, con una nota di Monica Bruni, Vicenza, Errepidueveneto, 1993); De itinere reginae Sabaeae, «Latinitas», XXX, 3, 1982, pp. 200-203 (poi, dopo due riprese in rivista e come plaquette, in Bandini 2007); Sancti duo decembris mensis (Nicolaus et Lucia), «Latinitas», XXXII, 4, I984, pp. 295-298 (poi, dopo due riprese parziali in Bandini 1986 e in rivista, in Santi di dicembre, cit.; Papiliones, «Latinitas», XXXIII, I, I985, pp. 58-68; Psyche, «Latinitas», XXXV, I, I987, pp. 63-67 (poi in Bandini 2007). Si veda la Bibliografia curata da Stefano Tonon in Bandini (2018: 693-704).

II Bandini (1989: I0): «Il suo appartarsi lontano dal centro non significa rinuncia al rapporto dialettico con la realtà e con la storia, è soltanto un modo di mettere a fuoco lo sguardo e rappresenta insieme la consapevolezza del "basso stato e frale" che i tempi concedono alla poesia».

I2 Bandini (1995: 76): «Da questo punto il luogo, inteso come luogo storico, diventa topos della poesia, cerca in stretti ambiti geografici, in aspetti consueti e familiari, l'universalità del proprio messaggio, un dilatato spazio comunicativo. Cesenatico è insieme, per Benzoni, luogo e topos della poesia».

I3 Bandini (2002b: 9): «Partendo da una "Liguria minima" [...] il pendolo della poesia si sposta verso un massimo di significazione. E questo quanto più tenace è il radicamento ai luoghi che costituiscono il suo mondo, quanto più angusti sembrano i confini di quella "contea di Levante" che egli ha descritto anni fa in bellissimi racconti».

I4 Più estesamente: «Anche quel tanto di ridotta "topicità", che è spesso proprio delle esperienze dialettali, può essere brulichio del cosmo». 
"topicità"» (Vicenza, non Aznèciv) ${ }^{15}$ è (era) l'espressione. Vale per Bandini, nei limiti temporali che ho delineato, quello che egli ha ben visto per la poesia trentina di Marco Pola:

Nella poesia dialettale Pola ha fissato il proprio destino esistenziale, la sua inquieta ricerca della verità, che tanto più è in grado di esprimere i propri valori universali quanto più sembra chiusa negli angusti confini di un atlante geografico-linguistico (Bandini 1975: 190).

Un secondo invito alla sperimentazione della scrittura dialettale sarà stato il rapporto tra dialetto e passato, un rapporto che - variamente declinato - è fondamentale per l'intera esperienza poetica di Bandini, che ha scritto:

ogni identità dei luoghi ha come base solida e rocciosa il mondo della memoria. E nessuna forma del comportamento, sia individuale sia collettivo, degli uomini è destinata ad affermarsi nel presente se non ha fatto i conti col proprio ieri. L'individualità è sempre una cosa che sta alle nostre spalle. Non è rassicurante, né è una qualche misura del conforto, come succede in alcune specie del classicismo. Ė una cosa con cui ci si misura con inquietudine, perché è un fatto esistenziale (il nostro essere qui, ora, col retaggio in noi di quegli embrioni del passato) ed è anche, insieme, sforzo e tensione della conoscenza (Bandini 1996: II).

Diremmo dunque del Bandini dialettale, assumendo come oggetto dello sguardo non il vissuto biografico ma il vissuto linguistico, ciò che egli scriveva del Raboni ultimo, quello di Barlumi di storia: «ll futuro (inteso come futuro possibile da realizzare in un concorde sforzo umano, ma anche come sgomento di fronte alle sue possibili, disumane incarnazioni) lo induceva a "voltarsi e guardare al passato" come l'angelo del romanzo di Thomas Clayton Wolfe» (Raboni 2006: I8). Questo Bandini, insomma, si fa indagatore dell'arcaicità linguistica in quanto essa «è portatrice anche di segnali per il futuro» (Bandini 1975: I89).

E c'è infine il dialetto come lingua dell'infanzia. Qui la sperimentazione di Bandini - nel senso della «rivisitazione», della «riappropriazione» dell'infanzia - si rispecchia nelle ricerche espressive di Bertolani e di Meneghello:

I5 Si veda Bandini (2008a: 20): nel nuovo «eone» Vicenza non è più un luogo che consenta la «pretesa laica di uno sguardo aperto su più vive vicende umane e su più vasti spazi del mondo»; è diventata «uno dei siti uguali tra loro e periferici del mondo. L'immagine della città non è più sentita come paradossale stimolo a essere, anche solo immaginandolo, alla sua pari. $\mathrm{E}$ da allora in qualche mia poesia [...] ho cominciato a chiamare la città col suo palindromo Aznèciv». 
La stessa rivisitazione dell'infanzia non è evasione nell'idillio ma necessità della mente prima che del cuore. L'occasione della poesia sarà l'inatteso ripresentarsi di una luce, il ripetersi di un gesto, che testimoniano che niente, malgrado la corsa del tempo, è cambiato e che compito del poeta è anche quello di perseguire questa perennità negli angoli dove si cela (Bandini 2002b: II).

Il dialetto non è [...], per Meneghello, soltanto la lingua della calda colloquialità, e nemmeno rappresenta il variegato ventaglio delle possibili espressività popolari. Il dialetto è la lingua che si riappropria dell'infanzia come momento centrale per decodificare il senso della vita (e la sua riposta poesia) (Bandini I987: VI).

Tutto ciò avviene, entro il lungo percorso poetico di Bandini, sotto l'imperio di una stringente «necessità», ma anche nel rifiuto di una sperimentazione perpetua, e soprattutto nella consapevolezza di quale debba essere la natura dell'impegno del poeta italiano nel nostro tempo - come si è visto. Di qui l'abbandono - o l'accantonamento - della scrittura in versi vicentini nel quindicennio successivo alla pubblicazione di Meridiano di Greenwich ${ }^{16}$.

3 Ho già trattato in altra sede (Zucco 2013) del rapporto che in Bandini si stabilisce tra infanzia e rima. Riprendo il discorso qui per mettere in luce come la sua riflessione sulla rima si svolga in una stretta implicazione con quella sul portato delle espressioni poetiche dell'infanzia (si è visto come la prima emergenza del dialetto nella sua poesia si dia come assunzione di una filastrocca dentro i versi in proprio); e passerò poi all'esame delle sue rime dialettali, così tornando al carattere sperimentale di questo settore della sua poesia.

L'insistenza più notevole nelle dichiarazioni di Bandini sulla rima sta nella sottolineatura del suo portato semantico:

La rima viene considerata, lo è stata in alcuni movimenti dell'avanguardia novecentesca, come un fatto costrittivo. E invece ha anche una funzione fondamentale: è risvegliatrice del subconscio. Essa fa improvvisamente avvertire parentele tra parole che nessuno avrebbe mai accostato fra loro. Ed è questa l'arcana forza della rima, che è forza soprattutto nella poesia della Divina commedia (Bandini 2002a: I29).

Si tratta, qui, della rima tout court. Rispetto alla rima nella poesia in lingua, quella nelle espressioni poetiche infantili sembra essere - suggerisce

I6 Non contengono poesie in dialetto le due brevi raccolte successive a Dietro i cancelli e altrove: Quattordici poesie (Bandini 2010) e Un altro inverno (Bandini 2012). 
Bandini - più trasparentemente rivelatrice dei suoi meccanismi a-logici: «l'irrazionalità, il non senso, il casuale sovrapporsi delle entità», che della rima costituiscono il tratto fondamentale, appaiono semplicemente «più evidenti» nelle «espressioni dialettali, come la conta e la filastrocca infantili» (Bandini 1983: 76). Nella sostanza, non sono forse riferibili alla rima tre affermazioni come le seguenti?

Il carattere preminente di questa lingua infantile è la capacità (magica) di evocare oggetti fuori da schemi logici prefissati, con una attenzione acutissima al materiale fonico (Bandini 1983: 77);

Il gusto di allineare parole grammaticalmente imparentate dalla desinenza [...] per raccoglierle quasi in una festa di famiglia, è apparentemente innocuo; esse, scomparsi nel paese perduto i loro referenti, si prestano l'una con l'altra i loro valori di significato, confondono l'una dentro l'altra i magici aloni semantici (Bandini 1983: 80-8I);

Le parole scomparse vengono evocate come in una seduta spiritica, si organizzano secondo l'aspetto della forma e del suono, si scambiano una con l'altra parte del proprio contenuto semantico (Bandini 1987: VIII).

Si arriva così a cogliere chiaramente come occorra vedere nella loro continuità la poesia dialettale di Bandini e la sua poesia italiana, segnatamente nelle fasi in cui essa faccia più ampio ricorso alla rima ${ }^{17}$. E dunque: non sarà stata anche l'attrazione per la rima a condurre Bandini verso la poesia in dialetto? Intendo dire: la scrittura in dialetto non sarà stata avvertita, anche, come una modalità per la sperimentazione di «rime inedite e in ogni caso non usuali» (Bandini 2009: 170)? Proverò a rispondere a partire da un passo del saggio già citato su Bertolani. Bandini trascrive Er viàgio: «Er viàgio g'è chì, / 'nti muréti a seco / 'nte l'arbièto... // Mée o fée - chì a posso / 'mprende mègio / viagiàndome 'nter chèe» (Bertolani 2002: 7I). Quindi commenta:

Ogni lingua ha una sua rima ricorrente con la parola cuore. Qui il dialetto della Serra permette di rimare cuore con miele e fiele (e il fatto che venga rimata è aperta con é chiusa è perfettamente legittimo nella tradizione italiana dai Siciliani in poi). Le rime del dialetto (soprattutto quelle dei dialetti periferici e appartati, mai usati come lingua letteraria, che i poeti dei nostri anni prediligono) le sentiamo echeggiare per la prima volta, sono preziose pepite rinvenute scavando le vene, finora mai esplorate, del logos materno (Bandini 2002b: IO).

I7 La questione andrebbe naturalmente approfondita attraverso uno studio diacronico. La mia impressione è che la fase di minore interesse per la rima si verifichi, in Bandini, nelle poesie de La mantide e la città. Sarebbe significativo, allora, se la scrittura dialettale iniziasse negli anni immediatamente successivi. 
In altre parole: il volgersi ad altro codice linguistico - com'è la scrittura in dialetto - fa sì che a «scambiarsi una con l'altra parte del proprio contenuto semantico» possano essere parole diverse da quelle chiamate - fonicamente - a scambiarselo entro il codice normalmente in uso (nel nostro caso, l'italiano). Si tratterà allora di verificare in che misura gli accostamenti rimici entro un dato corpus dialettale realizzino l'una o l'altra di queste due situazioni:

A) la traduzione delle parole dialettali in rima produce una rima italiana;

B) la traduzione delle parole dialettali in rima non produce una rima italiana.

Chiamerò rispettivamente $r A$ e $r B$ le rime rispondenti alle due situazioni traduttorie. Sono $r B$ mée : chèe e fée : chèe di Bertolani. Da esse deriva - al lettore di poesia italiana - uno choc cognitivo; esso è la conseguenza del fatto che solo entro questo codice linguistico «laterale» si dà scambio di contenuto semantico tra «miele»e «fiele» da una parte, «cuore» dall'altra.

Torniamo allora alle poesie in cui Bandini fa uso del dialetto. E cominciamo dalle tre di Memoria del futuro, analizzando per prima Farfalle e vischio:

II càrpene sul pra' se desmentega presto dell'omo ch'è passà, indigeno o foresto.

Resta farfalle ciare sull'erba a farse vanto e vischio da ciapare il giovane saranto.

L'inverno viene presto e nissùn savarà se mi che son passà so' indigeno o foresto.

È una $r A$ pra' I : passà 3 («prato»: «passato»); sono $r$ B ciare 5 : ciapare («chiare» : «prendere»), vanto 6 : saranto 8 («vanto» : «verdone») e savarà IO : passà II («saprà» : «passato»). Più difficile categorizzare presto : foresto (vv. 2-4 e 9-I2): il mio Devoto-Oli (I97I), per esempio, dà foresto «forestiero» come venetismo. Non c'è dubbio, comunque, che a predominare siano le $r B$. Non trascriverò per intero Mia madre cuciva tomaie, per la quale dovremo attribuire le categorie di $r A$ e $r B$ sia a coppie di parole vicentine sia a coppie formate da una parola vicentina e da una italiana. Qui gli inserti vicentini portano due $r A$ : martello 2 : bello 4 (perfetta solo all'occhio, giacché bello, nel contesto lingui- 
stico vicentino, ha /1/ di grado tenue) e conversare 2I : scoraiare 22. Ben quattro le rB: tomaie I : passaie 5 («tomaie» : «cespugli»), pra' 28 : carità 33 («prati» : «carità»), saltare 31 : crosare 32 («saltare»: «crocicchi»), tomaie 45 : maie 46 («tomaie» : «maglie»), a cui si aggiunge l'imperfetta (ma allitterante) lissia 48 :letizia 50 («liscivia»: «letizia»). Ed ecco Quello che è vietato:

Come mi tormentaste in gioventù maledetti, canaie! Perché sono nato in un tempo che non ha perdono se non per chi ga schei?

E sento in alto tra gli onàri osei fare tinno all'estate, ma le mie mani sono scorticate, le gambe tute rosse da le ortighe.

O mama, dighe, dighe che no i me copa, che i me lassa stare! Gli prometto di andare lontano dove non darò fastidio.

Là vivrò da privato, soffocherò borborigmi e rancori, farò quel che i vol lori senza fumi per la testa.

Ogni di mangerò la mia minestra con gli occhi dentro il piatto.

Conosco bene quello che è vietato, che no se pole avere.

Qui gli inserti dialettali producono le $r B$ schei 4 : osei 5 («soldi» : «uccelli») e ortighe 8 : dighe 9 («ortiche» : «di' loro»/«digli»), la $r A$ stare Io : andare II e una rima che a rigore andrebbe classificata come $r B$, rancori $\mathrm{I} 4$ : lori $\mathrm{I} 5$, anche se l'esito traduttorio è l'imperfetta «rancori : «loro». Guardando ora all'altro capo del percorso dialettale di Bandini, l'inserto in explicit di Rappresentazione della mia morte al tempo delle guerre in Medio Oriente, anche qui riscontriamo il portato di una $r B$ : disgelo 76 : putelo 84 («disgelo» : «bambino»).

Le venti poesie vicentine di Santi di Dicembre e Meridiano di Greenwich ${ }^{18}$ costituiscono un corpus abbastanza ampio da consentirci uno sguardo di insieme. I dati numerici sono assai chiari, ma richiedono la discussione preliminare di alcuni casi particolari. Va innanzitutto considerato che, come

I8 I due libri saranno citati, d'ora in avanti, anche nelle sigle SD e MG. 
nelle poesie italiane, anche in quelle vicentine Bandini fa uso di rime imperfette e di assonanze con funzione dichiaratamente rimica. Accostiamo allora alle $r A$ celeste : finestre (SD, I puteleti del vampiro, vv. 28-29), alle $r B$ campane «campane» ${ }^{19}$ : rame «rami» (SD, I puteleti del vampiro, vv. 9-Io), svèntola «ceffone»: méssola «falce»(SD, Desso i me spoia nuo, vv. 7-9), chichingeri «alchechengi» : in ghìngheri (SD, Vento e fogo, vv. 2I-22), ruma «rùfola» : su l'usma «in traccia» (MG, La ciupinara, vv. 3I-33), caco : almanco «almeno» (MG, El nome, vv. 33-35), de roèrso "per rovescio» : messo (v.) (MG, La ciupinara, vv. 42-43), tórte «prenderti» : note «notte» (MG, L'omo col saco, vv. 4-7), relòjo «orologio»: foje «foglie» (MG, L'omo col saco, vv. I2-I3 int.) e l'eccedente viole : sole : cotole "gonne» (SD, No volevo, vv. 6 int.-9 int.-II int.). C'è poi una serie di rime che a rigore andrebbero registrate come $r B$, ma che varie ragioni inducono ad accostare invece alle $r A$ : gli esiti traduttori di spia «spiano» : via (avv.) (MG, El nome, vv. 5-6) e bona «buona»: sona «suonano» (MG, L'anguria, vv. 7-9 int.) sono rime eccedenti; quelli di ale «ali» : temporale (MG, Oga Magòga, vv. I7-22) e rama «ramo» : ciama «chiamano» (MG, Oga Magòga, vv. 5-7) sono rispettivamente una rima imperfetta e una rima imperfetta eccedente. Nel caso di casoti : teremoti (MG, L'anguria, vv. I2-I3) ci troviamo di fronte a una $r A$ se per il primo rimante si accoglie la traduzione autoriale «capanni»; traducendo con l'accettabilissimo «casotti» avremmo una rima imperfetta per la diversa intensità consonantica. Quanto a mi : ti (MG, El nome, vv. 20-23), si tratta di una $r B$ solo in virtù di un'astrazione grammaticale (qui mi vale «io»); goldoni «preservativi» : balconi (MG, Spassaure, vv. Io int.-25) risulta una $r A$ recependo come italiano il settentrionalismo che è in prima posizione nella coppia. Un caso analogo è offerto da luna : cuna (SD, I puteleti del vampiro, vv. 37-39): Bandini traduce il secondo rimante con «culla», ma il significato del letterario e regionale cuna è trasparente.

Veniamo così ai dati numerici, che escludono tutte le coppie di cui ho appena trattato. Le serie rimiche (coppie e terne) del tipo $r A$ sono trentotto (ho escluso dal computo le rime identiche, e incluso sei rime interne) ${ }^{20}$;

I9 Metto tra caporali le traduzioni dell'Autore, tra apici le mie.

20 Per completezza: A: va : sa (SD, Drio de la porta, vv. I-5), sità «città»: va (SD, Sta lingua, vv. 19-20), stà «stato» : prà «prato» (MG, La ciupinara, vv. 63-65); AME: fame : luàme «letame» (MG, Spassaure, vv. I2-I5); ANDE: spande : grande (SD, La grande late, vv. 30-3I); ARE: fare : passare (SD, Vento e fogo, vv. I3-I4 int.); ficare «ficcare»: frugnare «frugare» (MG, La ciupinara, vv. 54-56), fumare : arfiare "ansimare» : segare (MG, Spassaure, vv. 20-2I-23 int.), guera "guerra» : tera «terra» (MG, Guera, vv. I-4); ARIA: aria : luna lunaria «luna calendaria» (SD, La grande late, vv. 27-29); ARME: salvarme «salvarmi» : masenarme «maciullarmi» (MG, Guera, vv. 2-I4); AVA: vendema$v a$ «vendemmiava» : sbimava «schiumava» : rivava 'arrivava' (MG, L'anfelo imbissà, vv. II-I6-I8); ECE: rece 'orecchie' («orecchi») : vece 'vecchie' (SD, Imbriago, vv. 4-5); EI: osèi «uccelli» : buèi 'budelli' («bucce di mortadella») : fradèi «fratelli» (MG, Spassaure, vv. 5-II int.-I4); ELE: matonele «mattonelle» : stele «stelle» (MG, Casa voda, vv. IO-I3); ENTE: sente : gente (MG, So' tel canton, vv. 6-7); ENTI: senti : denti (MG, El nome, vv. 3I-34); ERDE: verde : perde (SD, I puteleti del vampiro, vv. 3-5); ESTE: Este (toponimo) : celeste (SD, Vento e fogo, vv. 6-7); ESTA: festa : testa (SD, Imbriago, vv. 
quelle (sempre coppie e terne) del tipo $r B$ sono settantacinque (delle quali sedici interne). Il dato conferma dunque quanto si è visto nell'analisi delle tre poesie di Memoria del futuro. Il rimario del Bandini vicentino, per quel che riguarda le $r B$, è questo:

\section{A}

istà «estate» : robà «rubato» (MG, Casa voda, vv. I7-I8)

istà «estate» : scoltà «ascoltato» (MG, L'anguria, vv. I4-I5)

AI

mai : bài «insetti» (MG, La ciupinara, vv. 32-34)

gài «abbia» : mai (MG, El nome, vv. 8-Io)

\section{AJA}

passaja «siepe di cinta» : maja «maglia» (MG, Oga Magòga, vv. I8-2I)

ALO

palo 'palo' (segare el palo «tagliare la corda») : galo «gallo» (MG, Spassaure, vv. 23-26)

\section{AN}

organ «uragano» : de straman «di traverso» (SD, Vento e fogo, vv. IO-I4)

ANA

s-ciarana «schiarita» : lana (MG, Oga Magòga, vv. 19-23)

ANCA

man sanca «mano sinistra»: bianca (SD, La grande late, vv. I2-I4)

manca «mancano»: man sanca «mano sinistra» (SD, La grande late, vv. 33-34)

ANDA

da ogni banda «da ogni parte» : filanda (SD, Sta lingua, vv. 25-28)

da ogni banda «dappertutto» : granda «grande» (MG, L'omo col saco, vv. 22-25)

\section{ANE}

anguane «fate d'acqua» : altane (SD, Sta lingua, vv. 7-I6; anguane I7)

I-3), festa : resta (SD, No volevo, vv. IO-I2), testa : tempesta : festa (MG, Sagra, vv. 4-I3-I4); ETE: pirolete 'piroette' : macete 'macchiette' (SD, I puteleti del vampiro, vv. I8-20); IN: cavalin «cavallino»: putin «bambino» (SD, Vento e fogo, vv. 2-3); INA: aspirina : medissina «medicina» (MG, El nome, vv. I4-I5); IRO: in giro : vampiro (SD, I puteleti del vampiro, vv. I3-I5); O: so : go 'ho' (MG, El nome, vv. 25-26); OCI: genoci 'ginocchi' ("ginocchia») : oci 'occhi' (SD, Imbriago, vv. 8-II); OLE: viole : sole (SD, No volevo, vv. 6 int.-9 int.; segue cotole II int.); OLTA: n'altra volta : scolta «ascolta» (MG, El nome, vv. 29-30); ONA: parsona «persona» : bona 'buona' ("giusta») (SD, Vento e fogo, vv. I7-I8); ONDO: girotondo : mondo (SD, I puteleti del vampiro, vv. 3I-33); ORDO: sordo : ricordo (v.) (MG, El nome, vv. 24-27); ORTA: morta : porta (SD, Drio de la porta, vv. I8-I9); ORTI: orti : morti (SD, Sta lingua, vv. 32-35); OTE: grote «grotte» : mesanote «mezzanotte» (SD, Sta lingua, vv. 8-9); USI: Giusi : musi (MG, El nome, vv. 2-3); UTI: farabuti «farabutti» : tuti «tutti» (MG, Casa voda, vv. I-3). 
APE

frape «grinze» : cape «conchiglie» (MG, La ciupinara, vv. 59-60)

API

a s-ciapi «a stormi» : scapi «scappi» (SD, I puteleti del vampiro, vv. 4I-44)

ARA

sisara «nebbia ghiacciata» : ruara «solco di ruota» (SD, I puteleti del vampiro, vv. 50-5I) sisàra «brina gelata» : ciupinara «talpa» (MG, La ciupinara, vv. I2-I5)

ARE

sesolare «mietere» : desfare «disfare» (MG, Oga Magòga, vv. 13-20)

biscolare «dondolare» : mare «madre» (MG, Sagra, vv. I7-I9)

sercare «cercare» : crosare «crocicchi» (MG, Sagra, vv. 5-7)

ARI

pomari «meli» : botegari «negozianti» (SD, Sta lingua, vv. 24-3I int.)

ARO

luamaro «letamaio» : saresaro «ciliegio» (SD, Imbriago, vv. I2-I4)

armaro «armadio» : fogolaro «focolare» : granaro «solaio» (MG, Casa voda, vv. 6-8-18 int.)

ASSI

omenassi «omacci» : sassi «sassi» (SD, La grande late, vv. 5-8; poi omenassi 9)

ASSO

basso : tremasso «tremito» (SD, I puteleti del vampiro, vv. 27-30)

tremasso «tremore»: stramasso «materasso» : cadenasso «catenaccio» (MG, L'omo col saco, vv. I3 int.-I6-I8)

ATE

bate «battono» : tate «bambine» : late «latte» (SD, La grande late, vv. 35-36-39)

sate «zampe»: fate «fatte» (MG, La ciupinara, vv. 39-4I int.; fate «fatte» 50)

AVA

sbiava «sbiadita» : rivava «arrivava» (MG, La ciupinara, vv. I4-I5)

EGNA

vegna 'venga' («giunga») : insegna (v.) (MG, La ciupinara, vv. I6-I7)

EI

putèi «bambini» : dèi «dita» (SD, La grande late, vv. 2-4)

vedèi «vitelli»: putèi «bambini» (MG, Sagra, vv. I6-I8)

déi «dita»: putèi «bambini» (MG, L'anguria, vv. 5-6 int.) 


\section{ELE}

quele «quelle» (pron.) : putele «bambine» : campanele «campanule» (SD, No volevo, vv. 5-II-I4)

pomèle «bacche» : putèle «bambine» (agg.) (MG, La ciupinara, vv. 3 int.-8 int.)

stele (2) : in sfrasele «in frantumi» (MG, La ciupinara, vv. 36-37-40)

galinèle «valerianella» : satèle «zampine» (MG, La ciupinara, vv. 66-68)

\section{ENTE}

sente : da rente 'più vicino' (SD, La grande late, vv. 20-2I)

\section{ENTO}

drento 'dentro' : vento (MG, L'omo col saco, vv. 8-I4)

ERA

nera : palpièra «palpebra» : tera «terra» (MG, La ciupinara, vv. 49-5I-55)

\section{ERE}

masiere «muricce» : piere «pietre» (SD, Sta lingua, vv. IO-I3)

\section{ESE}

siése «siepi» : sirese «ciliegie» (MG, La ciupinara, vv. 2-6)

ETA

seleghéta «passera» : bachéta «bacchetta» (MG, Spassaure, vv. I3-I6 int.)

\section{ETO}

toseto «bambino» : t'imprometo «ti prometto» (MG, L'omo col saco, vv. 2-3)

toseto «bambino» : leto «letto» (MG, Casa voda, vv. I4-I5)

\section{IA}

pria «cote» : finia «finita» (SD, I puteleti del vampiro, vv. 23-25)

via (avv.) : bria «briglia» (SD, Vento e fogo, vv. I-8)

IGO

castigo (n.) : sigo «grido» (n.) (MG, So' tel canton, vv. 2-6 int.)

IN

camisolin «farsetto» : a delin «per il dito» : spanavin «erba acetosa» (SD, I puteleti del vampiro, vv. I7-I9-2I)

INA

puina «ricotta» : stamatina (SD, Imbriago, vv. 9-IO)

ISO

Treviso : griso «grigio» (SD, Vento e fogo, vv. 4-5) 


\section{ISOLE}

sgrìsole «brividi» : zisole «giuggiole» $(\mathrm{SD}$, La grande late, vv. 37-38)

ITO

dito «detto» (v.) : sofito «soffitto» (SD, Drio de la porta, vv. I4-I6)

IVO

bonorìvo «mattiniero» : vivo (v.) (MG, El nome, vv. 39-4I)

$\mathrm{O}$

pierò «Pierrot» : can buldò (SD, Carnevale, 3 int.-I3)

\section{OCHI}

in tochi «in pezzi» : ochi 'maschi dell'oca' [deventà ochi «istupiditi»] (SD, Vento e fogo, VV. II-I2)

\section{OCI}

oci «occhi»: coci «fiaccherai» (SD, Sta lingua, vv. 30-3I)

OGA

tóga «prenda» : Oga Magòga (MG, Oga Magòga, vv. II-I2 int.)

\section{OLE}

gole : vene latarole «vene da latte» (SD, I puteleti del vampiro, vv. 38-39 int.)

\section{OMBI}

bombi «madidi» : colombi (MG, Casa voda, vv. I6-I9)

ONA

parona «padrona» : dona «donna» (SD, I puteleti del vampiro, vv. 4-7)

ONTE

monte: scónte «stradette non frequentate» (MG, El nome, vv. 19-20)

OPE

falope «frottole» : strope «rami di salice» (SD, I puteleti del vampiro, vv. 32-35)

ORA

laora «lavorano»: fora 'fuori' (SD, La grande late, vv. IO-I3)

laóra «lavorano» : fóra 'fuori' (MG, La ciupinara, vv. 45-47)

ORE

lóre «loro» ('esse') : amore (MG, L'anguria, vv. Io int.-I7 int.)

ORO

lavoro (v.) : móro «muoio» (MG, El nome, vv. 40-42) 
OSE

nóse «nocche» : tose «ragazze» (MG, L'anguria, vv. 4-I5 int.)

OSI

$s c$-iosi «chiocciole»: strosi «sentieri» (SD, La grande late, vv. 26 int.-27 int.)

OSSA

grossa : giossa «goccia» (MG, Oga Magòga, vv. 2-3)

OTO

sangiòto «singhiozzo»: sóto «sotto» (MG, La ciupinara, vv. 67-69)

$\mathrm{U}$

turlulú «balordo» : più (MG, El nome, vv. 9-Io int.)

UA

bua «piccolo male» : pua «bambola» (SD, La grande late, vv. I6-I9)

URE

spassaùre «immondizie» : vansaùre «avanzi» (MG, Spassaure, vv. 3-I8; ripresa imperfettamente da scuro I9 int.)

URIA

scúria «frusta» : anguria (MG, L'omo col saco, vv. 2I-26)

Non c'è lo spazio per un'analisi semantica complessiva: dunque chiudo invitando il lettore alla ricerca delle «preziose pepite» contenute in questo elenco e all'immaginazione della gioia che Bandini deve aver provato trovando che nel suo dialetto letamaio rima con ciliegio, bambina con bacca, chiocciola con sentiero...

\section{Bibliografia}

Bandini, Fernando, In modo lampante, Venezia, Neri Pozza, 1962.

-. Per partito preso, Neri Pozza, [Venezia], 1965.

-. Memoria del futuro, Milano, Mondadori, 1969.

- Arcaicità e futuro nella poesia dialettale di Pola, in M. Pola, Cento poesie scelte 1936-1974, con saggi di Armando Balduino, F.B. e Andrea Zanzotto, Milano, All'Insegna del Pesce d'Oro, 1975, pp. 183-190.

-. La mantide e la città, Milano, Mondadori, 1979.

- Dialetto e filastrocca infantile in 'Libera nos a malo' e 'Pomo pero', in Su/Per Meneghello, a cura di Giulio Lepschy, Milano, Edizioni di Comunità, I983, pp. 73-83. 
-. Lo ferm voler q'el cor mintra..., «lengua», 5, 1985, pp. II7-II8 [entro la sezione: A. Giacomini - F.B., Da Arnaut Daniel, pp. III-I2I; alle pp. II9-I2I si legge la sestina di Arnaut nel testo critico e con la traduzione italiana (a pié di pagina) di Gianluigi Toja].

-. In lingue morte, con traduzione e nota dell'autore, «Almanacco dello Specchio», I2, a cura di Marco Forti; con la collaborazione di Giuseppe Pontiggia, Milano, Mondadori, 1986, pp. 227-240.

-. Introduzione, in Luigi Meneghello, Pomo pero. Paralipomeni di un libro di famiglia, Introduzione di F.B., Milano, Mondadori, I987, pp. V-IX.

-. Elogio della frontiera, in Eugenio De Signoribus, Case perdute, Ancona, Il lavoro editoriale, I989, pp. 5-II.

-. Figure e metri nella poesia di Marco Pola, in Poesia dialettale e poesia in lingua nel Novecento. Intorno all'opera di Marco Pola, Atti di seminario, Trento, ottobre 1993, a cura di Anna Dolfi, Milano, All'Insegna del Pesce d'Oro, I994, pp. 85-94.

- Benzoni o il coraggio della poesia, in Per Ferruccio Benzoni. Studi e testi (Biblioteca Comunale [di Cesenatico], 23 novembre I99I), a cura di Simonetta Santucci, xilografie di Luigi Berardi, Lugo, Associazione culturale «Il bradipo», 1995, pp. 70-78.

-. Piovene e Vicenza, in Guido Piovene tra idoli e ragione, Atti del Convegno di Studi, Vicenza, 24-26 novembre 1994, a cura di Stefano Strazzabosco, Venezia, Marsilio, 1996, pp. II-I8.

—. Meridiano di Greenwich, Milano, Garzanti, 1998 [Bandini 1998a].

- Le ragioni della poesia, in Le ragioni della poesia. Atti del convegno sull'opera di Fernando Bandini, a cura di Gabrio Vitali, Torre Boldone, Grafital, 1998, pp. 23-38 [Bandini 1998b].

-. Poesia d'eccezione, «Cooperazione», 43, 24 ottobre 200I, p. 72 [intervista a R. Zucco].

-. Il canto XXII del Purgatorio, in Regnum celorum vïolenza pate. Dante e la salvezza dell'umanità [Letture dantesche giubilari, Vicenza, ottobre 1999 - giugno 200o], a cura di Giuseppe Cannavò, Montella, Accademia Vivarium Novum, 2002, pp. I2I-I4I [Bandini 2002a].

-. Risillabare le parole ingenue, in Bertolani 2002: 7-I2 [Bandini 2002b].

-. Raboni primo e secondo, in Per Giovanni Raboni, Atti del Convegno di Studi, Firenze, 20 ottobre 2005, a cura di Adele Dei e Paolo Maccari, Roma, Bulzoni, 2006, pp. II-I8.

-. Dietro i cancelli e altrove, Milano, Garzanti, 2007.

-.Vicenza/Aznèciv: una città al cinema, il cinema di una città, in Vicenza e il cinema, a cura di Alessandro Faccioli, Venezia, Marsilio, 2008, pp. I8-20 [Bandini 2008a]. 
-. Vicenza o dellinattualità [conversazione con Roberto Galaverni, Massimo Raffaeli e Francesco Scarabicchi], «Alias», IX, 47 (534), 29 novembre 2008, pp. 20-22 [Bandini 2008b].

-. L'onore del poeta [2007], in Il Veneto che amiamo. Incontri con Fernando Bandini, Luigi Meneghello, Mario Rigoni Stern e Andrea Zanzotto, Prefazione di Goffredo Fofi, [s.l.] Edizioni dell'Asino, 2009, pp. I47-I83.

-. Quattordici poesie, con tre note di Pietro Gibellini, Massimo Raffaeli e Francesco Scarabicchi, Brescia, L’Obliquo, 2010.

-. Un altro inverno, Valverde (Catania), Il Girasole, 2012.

-. Tutte le poesie, a cura di Rodolfo Zucco, introduzione di Gian Luigi Beccaria, con un saggio biografico di Lorenzo Renzi, Milano, Mondadori, 2018.

Beccaria, Gian Luigi, Bandini, una tempra di grazia, in Bandini 2018: V-XX.

Bertolani, Paolo, Se de sea, Prefazione di Fernando Bandini, Genova, San Marco dei Giustiniani, 2002.

Mengaldo, Pier Vincenzo, Nota a "Festa d'inverno» [1976], ora in Id., Per Vittorio Sereni, Torino, Nino Aragno, 2013, pp. 243-267.

Segre, Cesare, Gli strati linguistici in «Libera nos a malo» di Meneghello, in Id., Intrecci di voci. La polifonia nella letteratura del Novecento, Torino, Einaudi, I99I, pp. 59-69.

Todoverto, Marco, L'«anema incateià» di Fernando Bandini: stile e metro delle poesie dialettali, Università degli Studi di Padova, Dipartimento di Studi Linguistici e Letterari, rel. Prof. Luca Zuliani, A.A. 2016/20I7.

Zucco, Rodolfo, "Certi poeti». Ipotesi sulla lingua poetica di Bandini, in Gli scrittori vicentini e la lingua italiana, a cura di Antonio Daniele, Vicenza, Accademia Olimpica, 2013, pp. 274-303. 